\title{
Validating and expanding the Baveno VI criteria for esophageal varices in patients with advanced liver disease: a multicenter study
}

\author{
Adonis A. Protopapas ${ }^{a}$, Theodora Mylopoulou ${ }^{b}$, Vasileios P. Papadopoulos ${ }^{c}$, Kiriaki Vogiatzi ${ }^{b}$, \\ Ioannis Goulis ${ }^{\mathrm{a}}$, Konstantinos Mimidis ${ }^{\mathrm{b}}$
}

Aristotle University of Thessaloniki, Thessaloniki; Democritus University of Thrace, Alexandroupolis; ENARGEIA

Medical Ltd, Xanthi, Greece

\section{Abstract}

aFourth Department of Internal Medicine, Aristotle University of Thessaloniki, Thessaloniki (Adonis A. Protopapas, Ioannis Goulis); ${ }^{\mathrm{b}}$ First Department of Internal Medicine, Democritus University of Thrace, Alexandroupolis (Theodora Mylopoulou, Kiriaki Vogiatzi, Konstantinos Mimidis); “"ENARGEIA” Medical Ltd, Xanthi (Vasileios P. Papadopoulos), Greece

Conflict of Interest: None

Correspondence to: Konstantinos Mimidis, Professor of Internal Medicine and Gastroenterology, $1^{\text {st }}$ Department of Internal Medicine, Democritus University of Thrace, Alexandroupolis, Greece, e-mail: kostasmimidis@hotmail.com

Received 3 May 2019; accepted 17 September 2019; published online 23 October 2019

DOI: https://doi.org/10.20524/aog.2019.0429

\section{Introduction}

Development of esophageal varices is a significant and common complication of portal hypertension in cirrhotic patients. In the past, guidelines recommended screening of every cirrhotic patient for esophageal or gastric varices [1]. However, the emergence of transient elastography (TE) as a screening tool for cirrhosis and clinically significant portal hypertension $(\mathrm{CSPH})$ has prompted physicians to search for noninvasive tools able to identify patients with varices without the need for endoscopy. A number of studies combined several noninvasive markers, such as platelet count (PLT), liver stiffness, spleen size, and spleen stiffness, to identify patients who can avoid screening endoscopy for esophageal varices [2-6]. 
These scientific endeavors culminated in the Baveno VI workshop, which issued new recommendations regarding the diagnosis and management of CSPH. According to these guidelines, patients with compensated advanced liver disease (cALD), characterized by liver stiffness $<20 \mathrm{kPa}$ in combination with a PLT $>150,000 / \mu \mathrm{L}$ are at very low risk of presenting highrisk varices (HRVs), a term used to define esophageal varices that need therapeutic intervention [7]. Therefore, in that specific group of patients, preventive endoscopy of the upper gastrointestinal tract can be avoided. Since the publication of these guidelines, there have been several studies that focused on validating these guidelines and even some that have tried to develop similar criteria. Amongst these, there was a study that successfully expanded the Baveno VI criteria by setting cutoff values at $110,000 / \mu \mathrm{L}$ for PLT and $25 \mathrm{kPa}$ for LSM [8].

In the present study, we evaluated liver stiffness measurements and PLT, as well as other clinical and laboratory findings, in an effort to further extend our knowledge in this field. Our aim was to validate the Baveno and expanded Baveno VI criteria and investigate whether a different set of criteria based on a combination of clinical and laboratory findings could achieve better outcomes in identifying cirrhotic patients who can avoid screening endoscopy for esophageal varices.

\section{Patients and methods}

\section{Study population}

Consecutive patients with cALD, derived from 2 centers, were enrolled in the study. The inclusion criteria specified a diagnosis of chronic liver disease and LSM $>12 \mathrm{kPa}$, while the exclusion criteria were the presence of splanchnic vein thrombosis, previous use of $\beta$-blockers, a history of esophageal varices, and acute hepatitis (viral or alcoholic). The study was conducted in accordance with the Helsinki declaration [9]. For each enrolled patient, parameters included in the Baveno VI workshop criteria were collected, as well as a number of other clinical and laboratory parameters, such as cause of cirrhosis (chronic viral hepatitis $\mathrm{B} / \mathrm{C}$, alcohol, non-alcoholic fatty liver disease, etc.), Child-Pugh score, liver stiffness measurement (original data and logarithmic transformation), PLT, age, and sex. The parameters mentioned above were correlated with the presence and severity of endoscopically documented esophageal varices. Varices $\geq F 2$ in size, gastric varices, and varices with red spots were considered to qualify as HRVs. To achieve optimal correlation between endoscopic, laboratory and LSM data, the tests for every patient were conducted within a 6-month period.

\section{TE}

TE was conducted using Fibroscan ${ }^{\circledR}$ (Echosens, Paris, France). A total of 10 valid measurements were obtained from each patient using an M-size probe. The measurements were required to have $\geq 60 \%$ success rate and an interquartile range to median ratio $\leq 30 \%$, compatible with what is proposed by international guidelines [10-12].

\section{Statistical analysis}

Chi-square test was used for comparisons between expected and observed frequencies, while McNemar's test was used to determine any differences in a dichotomous dependent variable between the 2 related groups (specifically, to compare the diagnostic accuracy measures of the different criteria used to evaluate HRVs). In the case of residuals smaller than 5, Fisher's exact test was used. Student's $t$-test was used for comparison of continuous variables between 2 groups. The Levene test was used for comparison of variances between groups. Analysis of variance was used for comparison of continuous variables between 3 or more groups. In cases where the Levene test was statistically significant, either the Dunnett C or the Scheffé test was used to discriminate differences between groups. Otherwise, the LSD test was used. Binary logistic regression was used to predict the relationship between independent predictors and the dependent variables, where the dependent variable is binary. Receiver operating characteristic (ROC) curves were used to evaluate the diagnostic ability of binary variables; Youden's J index (sensitivity + specificity -1) was used for estimation of the best cutoff. Sensitivity, specificity, positive predictive value (PPV) and negative predictive value (NPV) were calculated and expressed with 95\% confidence intervals $(\mathrm{CI})$. Mean values $(\mathrm{N})$ are given with at least 2 significant digits and are accompanied by their standard errors (SE). The level of statistical significance was set to $\mathrm{P}<0.05$. The Bonferroni correction was applied when multiple comparisons were performed simultaneously, limiting the level of statistical significance to $\mathrm{a}=0.05 / \mathrm{m}$; $\mathrm{m}$ denotes the number of comparisons performed. All statistical analyses were supported by the SPSS 20.0 software package (IBM Corp. ( )).

\section{Results}

One-hundred seven consecutive patients with cALD, 65 men and 42 women, $63.7 \pm 1.2(30-87)$ years old, were enrolled in the study. All patients underwent screening esophagogastroduodenoscopy (EGD) and TE (TE).

Regarding the underlying cause of cALD, 26 (24.3\%) had a history of alcoholism, 49 (45.8\%) of chronic viral hepatitis B/C, 23 (21.5\%) of other clinical conditions (including nonalcoholic fatty liver disease, autoimmune hepatitis, primary biliary cirrhosis etc.), while 11 (10.3\%) had cryptogenic cirrhosis; 2 patients had multiple causes. For the severity of the liver disease, LSM revealed a mean value of $30.7 \pm 1.71 \mathrm{kPa}$ $\left(\log _{10}: 1.43 \pm 0.02\right)$ and a mean Child-Pugh score of $5.69 \pm 0.15$ (Child-Pugh A: 87.4\%, Child-Pugh B: 9.2\%, Child-Pugh C: $3.4 \%$; data refer to 87 patients) was observed. PLT was $129.0 \pm 6.3 \times 10^{3} / \mu \mathrm{L}$. Twenty-two patients (20.5\%) exhibited 
esophageal varices needing therapeutic intervention (F2/F3: group A), 34 patients (31.8\%) had small varices without red spots (F1: group B) while 51 (47.7\%) had portal gastropathy or normal endoscopic findings (F0: group C). A summary of the descriptive data is presented in Table 1.

Table 1 Descriptive statistics of patients enrolled in the study $(n=107)$

\begin{tabular}{|c|c|c|}
\hline Parameter & N (\%) & Mean \pm SD \\
\hline $\begin{array}{l}\text { Presence of varices } \\
\text { Portal gastropathy or normal } \\
\text { endoscopic findings (Group A) } \\
\text { Small varices without red spots } \\
\text { (Group B) } \\
\text { Esophageal varices needing } \\
\text { therapeutic manipulation } \\
\text { (Group C) }\end{array}$ & $\begin{array}{l}51(47.7) \\
34(31.8) \\
22(20.6)\end{array}$ & \\
\hline $\begin{array}{l}\text { Sex } \\
\text { Male } \\
\text { Female }\end{array}$ & $\begin{array}{l}65(60.7) \\
42(39.3)\end{array}$ & \\
\hline Age & & $63.7 \pm 12.1$ \\
\hline $\begin{array}{l}\text { Cirrhosis causes }^{\star} \\
\text { Alcohol } \\
\text { Viral hepatitis } \\
\text { Other (NASH, AI, PBC) } \\
\text { Cryptogenic }\end{array}$ & $\begin{array}{l}26(24.2) \\
49(45.8) \\
23(21.5) \\
11(10.3)\end{array}$ & \\
\hline LSM (kPa) & & $30.7 \pm 17.7$ \\
\hline $\log _{10} \mathrm{LSM}$ & & $1.43 \pm 0.23$ \\
\hline $\operatorname{PLT}\left(\times 10^{3} / \mu \mathrm{L}\right)$ & & $129.0 \pm 65.6$ \\
\hline $\begin{array}{l}\text { Baveno VI criteria met } \\
\text { Yes } \\
\text { No }\end{array}$ & $\begin{array}{l}13(12.1 \%) \\
94(87.9 \%)\end{array}$ & \\
\hline $\begin{array}{l}\text { Expanded baveno criteria met } \\
\text { Yes } \\
\text { No }\end{array}$ & $\begin{array}{l}30(28.0 \%) \\
77(72.0 \%)\end{array}$ & \\
\hline $\begin{array}{l}\text { PLT } / \log _{10} \text { LSM cutoff } 122,000 \text { met } \\
\text { Yes } \\
\text { No }\end{array}$ & $\begin{array}{l}23(21.5 \%) \\
84(78.5 \%)\end{array}$ & \\
\hline $\begin{array}{l}\text { PLT/ } \log _{10} \text { LSM cutoff } 92,000 \text { met } \\
\text { Yes } \\
\text { No }\end{array}$ & $\begin{array}{l}48(44.9 \%) \\
59(55.1 \%)\end{array}$ & \\
\hline
\end{tabular}

${ }^{*} \mathrm{~N}(\%)$ exceeds $100 \%$ as multiple causes were observed in 2 patients LSM, liver stiffness measurement; PLT, platelets
Comparing patients needing treatment to patients not needing treatment, no difference was found in age $(\mathrm{P}=0.219)$, sex $(P=0.504)$, cause of cirrhosis $(P=0.716)$, Child-Pugh stage $(\mathrm{P}=0.297)$, Child-Pugh score $(\mathrm{P}=0.398)$, liver stiffness measurement $(\mathrm{P}=0.093)$, or the logarithm of liver stiffness $(\mathrm{P}=0.081)$. However, PLT values differed between groups: patients not needing treatment had $138.5 \pm 7.4 \times 10^{3} / \mu \mathrm{L}$ while patients needing treatment presented lower PLT $\left(92.2 \pm 7.9 \times 10^{3} / \mu \mathrm{L}\right)$ $(\mathrm{P}=0.003)$. These values are presented in Table 2 .

\section{Validation of Baveno VI and expanded-Baveno VI criteria in the present study}

In an effort to validate the Baveno VI criteria in our patients, we found that 13 of them had PLT values $>150,000 \mu \mathrm{L}$ and liver fibrosis $<20 \mathrm{kPa}$, while 94 did not. Of the 13 patients who met the Baveno VI criteria, none needed treatment based on endoscopic findings, while of the 94 patients who did not meet the Baveno VI criteria, 22 needed treatment, as proven by EGD (1-sided $\mathrm{P}=0.041,2$-sided $\mathrm{P}=0.066$ ). In our study, the absence of the Baveno criteria predicted the detection of HRVs with $100 \%$ sensitivity, $15 \%$ specificity, $23 \%$ PPV, and 100\% NPV; by implementing the Baveno VI criteria, $12.1 \%$ of our patients would have avoided screening endoscopy.

Regarding the implementation of the expanded Baveno criteria to our study population, there were 30 patients who met the criteria and 77 who did not $(n=107)$. Of the 30 patients who met the expanded Baveno VI criteria, 1 needed treatment based on endoscopic findings, while of the 77 patients who did not meet the expanded-Baveno criteria, 21 needed treatment, as proven by EGD $(\mathrm{P}=0.006)$. The absence of the expanded Baveno criteria achieved $96 \%$ sensitivity, $34 \%$ specificity, $27 \%$ PPV and 97\% NPV for detection of HRVs. By implementing the expanded Baveno VI criteria, $28.0 \%$ of the patients would have avoided EGD, one of them (3.3\%) erroneously (Fig. 1).

\section{New approaches for HRV detection: the role of PLT/ $\log _{10}$ LSM ratio}

In relation to the above results, we further proposed new parameters of substantial prognostic value regarding the detection of HRV, involving PLT, LSM, and age values (absolute,

Table 2 Characteristics of patients who needed treatment versus patients who did not

\begin{tabular}{lccc}
\hline Parameter & $\begin{array}{c}\text { Varices not needing treatment } \\
(\mathrm{F} 0 / 1)(\mathrm{n}=85)\end{array}$ & $\begin{array}{c}\text { Varices needing treatment } \\
(\mathrm{F} 2 / 3)(\mathrm{n}=22)\end{array}$ & $\begin{array}{c}\text { P-value } \\
\end{array}$ \\
\hline Sex (male/female) & $53 / 32$ & $12 / 10$ & 0.504 \\
Cause of cirrhosis (viral/alcohol/other/cryptogenic) & $40 / 21 / 19 / 7$ & $9 / 5 / 4 / 4$ & 0.716 \\
Child-Pugh stage (A/B/C) & $62 / 5 / 3$ & $14 / 3 / 0$ & 0.297 \\
Age & $62.9 \pm 1.2$ & $66.5 \pm 1.8$ & 0.219 \\
Liver stiffness measurement $(\mathrm{kPa})$ & $29.2 \pm 1.8$ & $36.3 \pm 4.2$ & 0.093 \\
Platelets $\left(\times 10^{3} / \mu \mathrm{L}\right)$ & $138.5 \pm 7.4$ & $92.2 \pm 7.9$ & 0.003 \\
\hline
\end{tabular}

Continuous variables are expressed as mean \pm standard error 

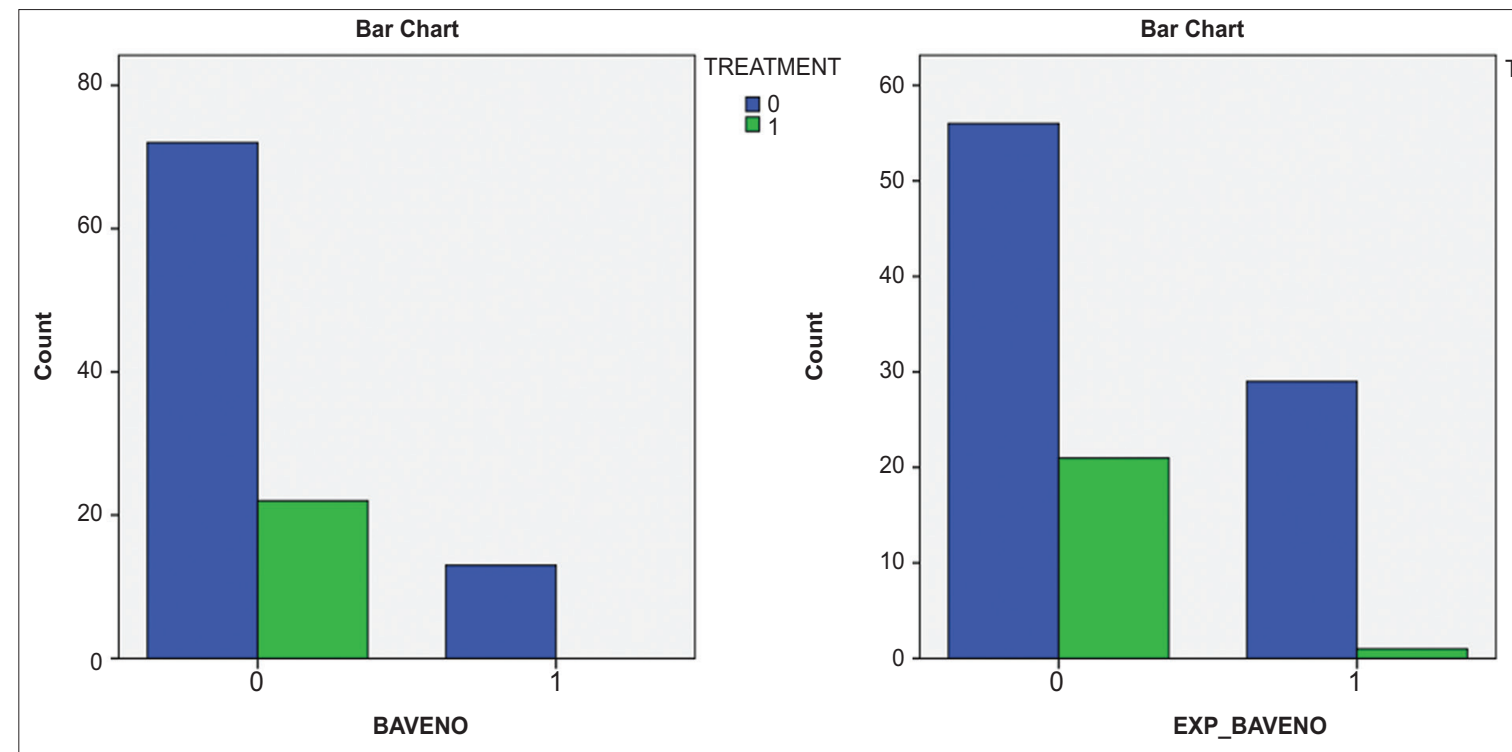

TREATMENT

$P=0.006$ (2-sided), $P=0.041$ (1-sided)
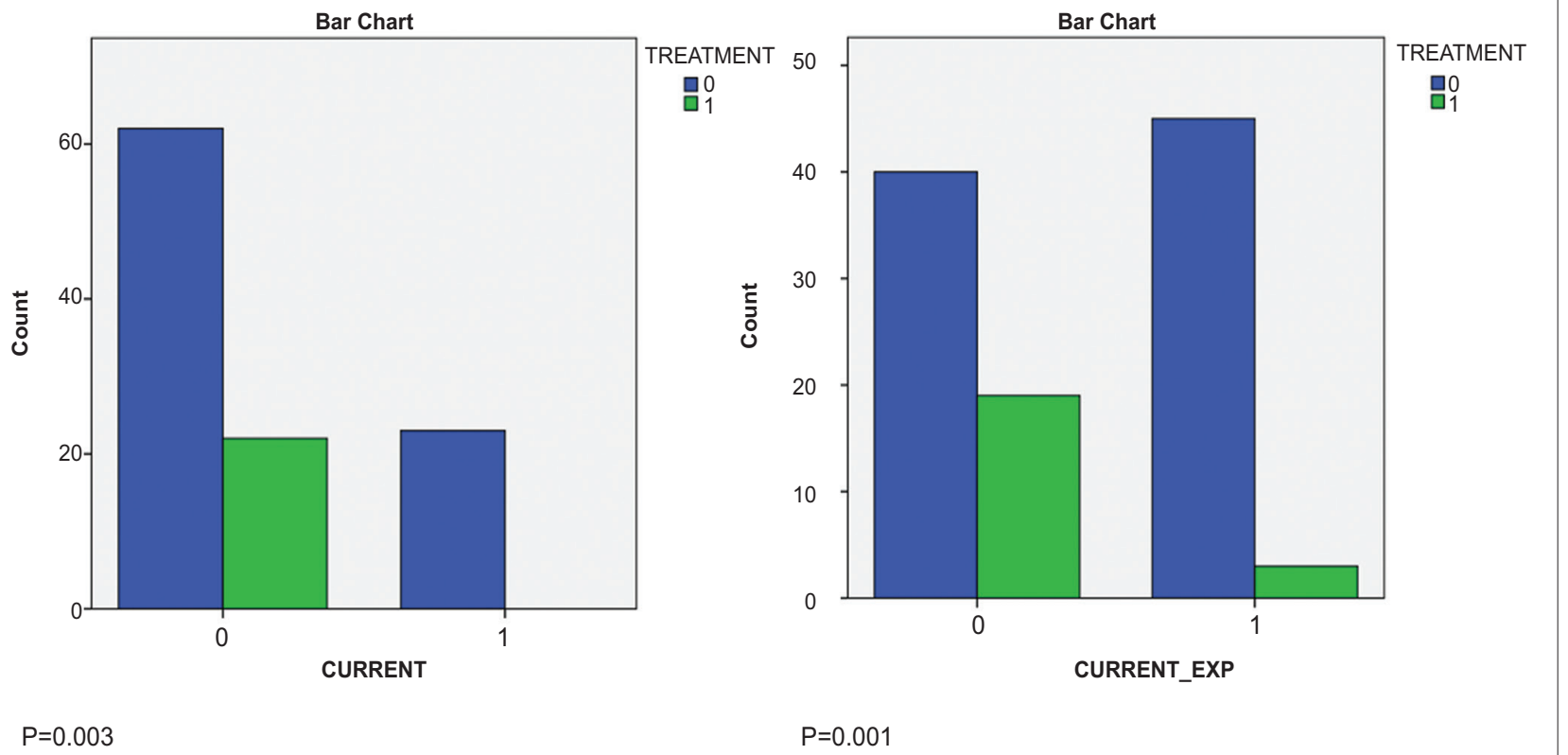

$P=0.003$

$\mathrm{P}=0.001$

Figure 1 Evaluation of Baveno (top left), expanded Baveno (top right), current (bottom left) and expanded current (bottom right) criteria based on data of the present study. Bars on the left side of charts refer to patients where criteria are absent, while bars on the right side of charts refer to patients where criteria are met (blue: patients not needing treatment; green: patients needing treatment)

logarithmic or inverse). These parameters were evaluated by univariate analysis and are presented, along with all detailed data, in Table 3. Furthermore, a binary regression model including these parameters gave a significance of $\mathrm{P}<0.05$ in multivariate analysis (with the exclusion of PLT in order to avoid collinearity due to the concomitant presence of $\log _{10}$ PLT). From both univariate and multivariate analyses, the ratio PLT $/ \log _{10} \mathrm{LSM}$ proved to be the most informative. Additionally, relevant ROC curves were plotted (Fig. 2). The area under the curve (AUC) and the statistical significance level for each curve were computed, as shown in Table 4. The results indicate that the ratio PLT/ $\log _{10}$ LSM exhibited the larger AUC (0.726) and the highest level of statistical significance ( $\mathrm{P}=0.001$ ) (Fig.3). PLT/ $\log _{10} \mathrm{LSM}$ $\leq 122,000 \mu \mathrm{L}^{-1} \mathrm{x} \mathrm{kPa}^{-1}$ (Youden's $\mathrm{J}=0.271$ ) predicted the detection of HRV with $100 \%$ sensitivity, $26 \%$ specificity, $27 \%$ PPV and $100 \%$ NPV $(\mathrm{P}=0.003)$. By adopting this cutoff, $20.6 \%$ of our patients would have avoided screening EGD, as none of the 22 patients who met the abovementioned criterion needed treatment based on endoscopic findings, while of the remaining 85 patients 23 needed treatment, as proven by EGD. This was a statistically significant improvement in the number of endoscopies avoided $(\mathrm{P}=0.006)$, compared to the application of the Baveno criteria.

In search of less strict criteria, we set the cutoff at $92,000 \mu \mathrm{L}^{-1}$ $\mathrm{x} \mathrm{kPa}^{-1}$, the value that gave the curve with the best possible 
Table 3 New parameters proposed for validation of alternative criteria to Baveno VI. Comparison between patients who needed treatment and those who did not

\begin{tabular}{|c|c|c|c|c|c|}
\hline \multirow[t]{2}{*}{ Parameter } & \multicolumn{2}{|c|}{ Mean \pm SE } & \multirow[t]{2}{*}{ Levene P } & \multirow[t]{2}{*}{ Univariate $\mathrm{P}$ (t-test) } & \multirow[t]{2}{*}{ Multivariate $\mathrm{P}$ (binary regression) $)^{* * *}$} \\
\hline & $\mathrm{F} 0 / 1$ & $\mathrm{~F} 2 / 3$ & & & \\
\hline LSM $(\mathrm{kPa})$ & $29.2 \pm 1.8$ & $36.3 \pm 4.2$ & 0.217 & $0.093^{* *}$ & \\
\hline $\log _{10} \mathrm{LSM}$ & $1.41 \pm 0.02$ & $1.50 \pm 0.05$ & 0.636 & $0.081^{* *}$ & \\
\hline $\operatorname{PLT}\left(\times 10^{3}\right)$ & $138 \pm 7.4$ & $92.1 \pm 7.9$ & 0.099 & $0.003^{*}$ & \\
\hline $\log _{10}$ PLT & $5.11 \pm 0.20$ & $4.93 \pm 0.19$ & 0.908 & $0.001^{*}$ & 0.328 \\
\hline $\mathrm{AGE}^{-1}$ & $0.0164 \pm 0.0004$ & $0.0154 \pm 0.0007$ & 0.444 & $0.255^{* *}$ & \\
\hline PLT/LSM & $6351 \pm 553$ & $3326 \pm 510$ & 0.055 & $0.008^{\star *}$ & 0.294 \\
\hline PLT/ $\log _{10}$ LSM & $102161 \pm 6303$ & $62770 \pm 5895$ & 0.074 & $0.003^{*}$ & 0.166 \\
\hline PLT/AGE & $2158 \pm 108$ & $1716 \pm 222$ & 0.914 & $0.069^{* *}$ & 0.406 \\
\hline PLT/(LSM x AGE) & $96.6 \pm 7.7$ & $53.1 \pm 8.7$ & 0.041 & $0.0004^{*}$ & 0.506 \\
\hline PLT/( $\log _{10}$ LSM x AGE) & $1577 \pm 88$ & $1018 \pm 115$ & 0.080 & $0.003^{*}$ & 0.179 \\
\hline
\end{tabular}

Continuous variables are expressed as mean \pm standard error (Mean \pm SE)

* Parameters remaining statistically significant after Bonferroni correction $(\mathrm{m}=10)$, setting a to 0.005

** Parameter excluded from statistical significance after Bonferroni correction

*** $\mathrm{P}<0.001$, Nagelkerke $\mathrm{R}^{2}=0.199$ for the whole model

LSM, liver stiffness measurement; PLT, platelets

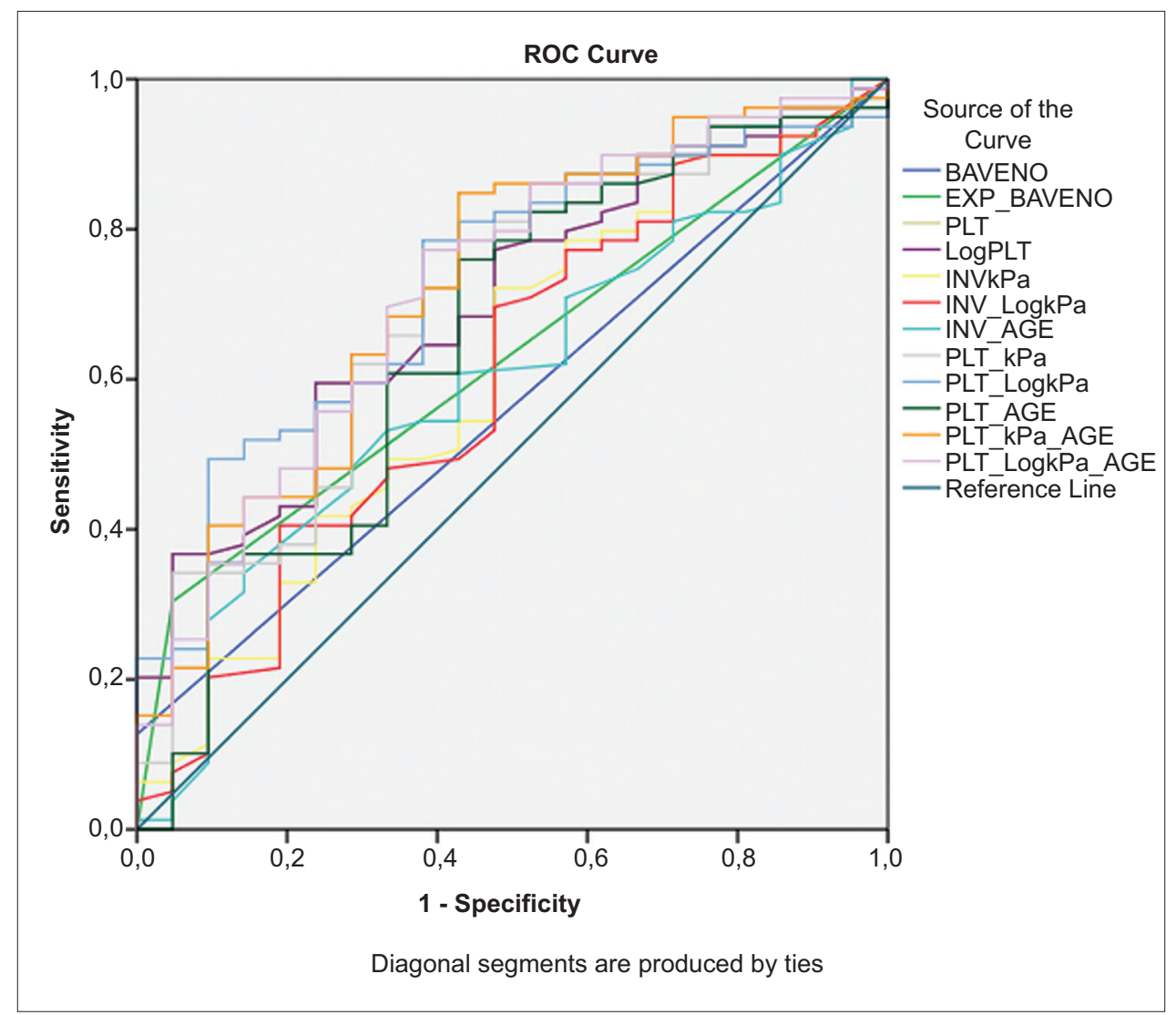

Figure 2 New parameters proposed for validation of alternative criteria to Baveno VI ones: ROC curve

BAVENO, Baveno criteria; EXP_BAVENO, expanded Baveno criteria; PLT, platelets; INV, inverse; $k P a$, liver stiffness measurement 
Table 4 New parameters proposed for validation of alternative criteria to Baveno VI: data from receiver operating characteristic curves

\begin{tabular}{|c|c|c|c|c|c|}
\hline \multirow[t]{2}{*}{ Variable } & \multirow[t]{2}{*}{ AUC } & \multirow[t]{2}{*}{ SE } & \multirow[t]{2}{*}{$\mathrm{p}$} & \multicolumn{2}{|c|}{ Asymptotic $95 \%$ confidence interval } \\
\hline & & & & Lower bound & Upper bound \\
\hline Baveno & 0.563 & 0.066 & 0.374 & 0.434 & 0.693 \\
\hline Expanded Baveno & 0.628 & 0.061 & 0.071 & 0.508 & 0.748 \\
\hline PLT & 0.698 & 0.060 & 0.005 & 0.581 & 0.815 \\
\hline $\log _{10}$ PLT & 0.698 & 0.060 & 0.005 & 0.581 & 0.815 \\
\hline $\mathrm{LSM}^{-1}$ & 0.608 & 0.071 & 0.131 & 0.469 & 0.746 \\
\hline $\log _{10} \mathrm{LSM}^{-1}$ & 0.602 & 0.071 & 0.154 & 0.462 & 0.741 \\
\hline $\mathrm{AGE}^{-1}$ & 0.596 & 0.067 & 0.176 & 0.465 & 0.728 \\
\hline PLT/LSM & 0.698 & 0.064 & 0.005 & 0.572 & 0.824 \\
\hline PLT $/ \log _{10}$ LSM & 0.726 & 0.058 & 0.001 & 0.612 & 0.840 \\
\hline PLT/AGE & 0.655 & 0.071 & 0.030 & 0.517 & 0.793 \\
\hline PLT/(LSMxAGE) & 0.722 & 0.063 & 0.002 & 0.598 & 0.845 \\
\hline PLT/( $\log _{10}$ LSMxAGE) & 0.721 & 0.062 & 0.002 & 0.599 & 0.843 \\
\hline
\end{tabular}

LSM, liver stiffness measurement; PLT, platelets; AUC, area under the curve; SE, standard error

Youden's index $(\mathrm{J}=0.438)$. Under these circumstances, PLT/ $\log _{10} \mathrm{LSM} \leq 92,000 \mu \mathrm{L}^{-1} \times \mathrm{kPa}^{-1}$ predicted the detection of HRV with $86 \%$ sensitivity, $53 \%$ specificity, $32 \%$ PPV and $94 \% \mathrm{NPV}$ $(\mathrm{P}=0.001)$. By adopting this cutoff, $44.9 \%$ of our patients would avoid screening EGD. However, 3 of the 48 patients (6.3\%) who met the abovementioned criterion needed treatment based on endoscopic findings, while of the remaining 59 patients, 19 needed treatment as proved by EGD (Fig. 1). The diagnostic accuracy measures of all tests are presented together in Table $5 \mathrm{a}$ and a comparison between them in Table $5 \mathrm{~b}$.

\section{Discussion}

We successfully validated the Baveno VI criteria in our study, showing that patients who met the criteria could safely avoid screening EGD, since none of them had HRVs. Although the criteria showed good sensitivity and NPV, only a small percentage of the study population could avoid EGD on that basis, while the majority of the patients did not demonstrate HRVs in EGD. Thus, we proposed new criteria, based on PLT, age, and LSM; among these, age proved to be non-informative. By using the ratio PLT/ $\log _{10} \mathrm{LSM}$ and setting the cutoff at $122,000 \mu \mathrm{L}^{-1} \times \mathrm{kPa}^{-1}$, we maintained optimal sensitivity and NPV, while achieving a substantial increase in the percentage of patients who could avoid EGD when compared with the Baveno criteria (18.2\% vs. $12.1 \%)$.

The Baveno VI criteria have been widely studied since they were published and many studies have validated their use [13-16]. The common conclusion of these studies is that the Baveno criteria can be used to exclude patients from EGD, with $<5 \%$ of these patients being at risk of having HRVs. However, what many studies stress is that the criteria may be too strict and in the process of excluding the least amount of cirrhotics

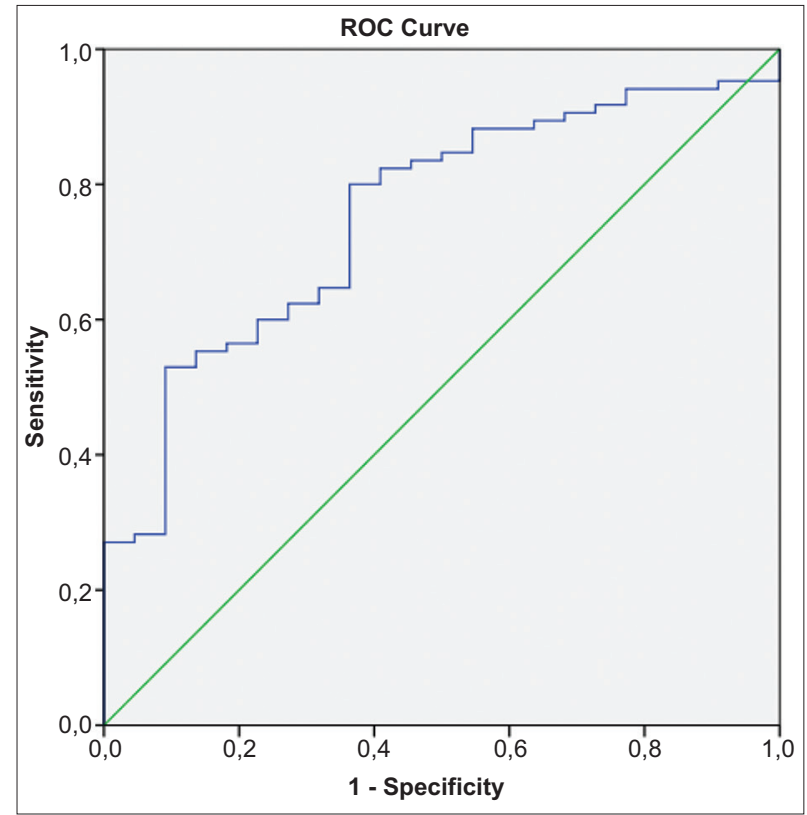

Figure 3 ROC curve for PLT / $\log _{10}$ LSM

LSM, liver stiffness measurement; PLT, platelets

with HRVs from screening EGD, fail to decrease the number of screening EGDs appreciably. Consequently, there have been many studies proposing alterations to the Baveno VI criteria, in order to maximize their efficacy. The most significant among these is a study that proposed expanding the Baveno criteria to include patients with PLT $>110,000 / \mu \mathrm{L}$ and $\mathrm{LSM}<25 \mathrm{kPa}$ [8]. This study succeeded in significantly decreasing the number of EGDs while keeping within the criteria a meager percentage of patients with HRVs. By applying these criteria to our study population, we achieved very satisfying sensitivity and NPV, while $28.0 \%$ of the patients would avoid EGD, though $3.3 \%$ of 
Table 5 (A) Diagnostic accuracy measures (with 95\% confidence intervals) of criteria used for evaluation of high-risk varices

\begin{tabular}{lcccc}
\hline & Sensitivity & Specificity & PPV & NPV \\
\hline Baveno VI & $100 \%$ & $15.29 \%$ & $23.4 \%$ & $100 \%$ \\
& $(84.56-100 \%)$ & $(8.4-24.73 \%)$ & $(21.82-25.06 \%)$ & \\
Expanded Baveno VI & $95.45 \%$ & $34.12 \%$ & $27.27 \%$ & $96.67 \%$ \\
& $(77.16-99.88 \%)$ & $(24.18-45.2 \%)$ & $(23.89-30.94 \%)$ & $(80.69-99.51 \%)$ \\
Study criteria $\left(122,000 \mu \mathrm{L}^{-1} \mathrm{x} \mathrm{kPa}^{-1}\right)$ & $100 \%$ & $26.19 \%$ & $27.06 \%$ & $100 \%$ \\
& $(85.18-100 \%)$ & $(17.2-36.93 \%)$ & $(24.62-29.65 \%)$ & $32.2 \%$ \\
Study criteria $\left(92,000 \mu \mathrm{L}^{-1} \mathrm{x} \mathrm{kPa}^{-1}\right)$ & $86.36 \%$ & $52.94 \%$ & $(26.42-38.59 \%)$ & $(83.72-97.77 \%)$ \\
\hline
\end{tabular}

$\overline{P P V}$, positive predictive value; $N P V$, negative predictive value

Table 5 (B) McNemar's tests for diagnostic accuracy measures of Table 5A

\begin{tabular}{lcccc} 
& Baveno VI & Expanded Baveno VI & $\begin{array}{c}\text { Study criteria } \\
\left(122,000 \mu \mathrm{L}^{-1} \times \mathrm{kPa}^{-1}\right)\end{array}$ & $\begin{array}{c}\text { Study criteria } \\
\left(92,000 \mu \mathrm{L}^{-1} \times \mathrm{kPa}^{-1}\right)\end{array}$ \\
\hline Baveno VI & & & $0.006^{*}$ & $<0.001^{*}$ \\
Expanded Baveno VI & $<0.001^{*}$ & - & 0.143 & $<0.001^{*}$ \\
Study criteria $\left(122,000 \mu \mathrm{L}^{-1} \mathrm{x} \mathrm{kPa}^{-1}\right)$ & $0.006^{*}$ & 0.143 & - & $<0.001^{*}$ \\
Study criteria $\left(92,000 \mu \mathrm{L}^{-1} \times \mathrm{kPa}^{-1}\right)$ & $<0.001^{*}$ & $<0.001^{*}$ & $<0.001^{*}$ & - \\
\hline
\end{tabular}

${ }^{*}$ Parameters remaining statistically significant after Bonferroni correction $(\mathrm{m}=6)$, setting $\alpha$ to 0.00833

them erroneously. These data show a further reduction in the number of EGDs with a reasonable loss amount.

Inspired by this paradigm, we searched for potent alternative cutoffs for our criteria. Therefore, we investigated every single curve point, starting from the one that achieved the best possible Youden's index ( $J=0.438$ ), namely $92,000 \mu \mathrm{L}^{-1} \times \mathrm{kPa}^{-1}$. Indeed, PLT/ $\log _{10} \mathrm{LSM} \leq 92,000 \mu \mathrm{L}^{-1} \times \mathrm{kPa}^{-1}$ significantly raised the specificity of the criteria. By adopting this cutoff, almost half of our patients would avoid screening EGD. However, a significant percentage of the patients who met this criterion (6.3\%) had HRVs.

A subject of debate that has been raised by previous studies is the percentage of missed HRVs that we consider acceptable when evaluating patients using noninvasive techniques. The concept raised by the authors of the study that heavily influenced the Baveno VI criteria, titled "the Anticipate study", was that a percentage as high as $5 \%$ can be considered acceptable [2]. This concept has been adopted by other researchers and even included in a technical review of the American Gastroenterological Association regarding the role of $\mathrm{TE}$ in chronic liver diseases $[11,17]$. Under this view, our expanded criteria, using a cutoff of $92,000 \mu \mathrm{L}^{-1} \times \mathrm{kPa}^{-1}$, could tolerate a slightly elevated percentage of false negatives $(6.3 \%)$ to achieve an auspicious amount of screening avoidance (44.9\%). Furthermore, taking into account that the percentages described are very close to the $5 \%$ threshold, that the expanded Baveno VI criteria showed only a $1.6 \%$ risk of not identifying HRVs in the parent study, and that both offer a significant advantage in terms of EGD avoided, their use should not be summarily rejected.

Primary limitations of our study are the relatively small sample size and the limited number of parameters examined. Other studies have also used additional parameters to the ones used in the Baveno VI criteria. As a case in point, a study by

\section{Summary Box}

\section{What is already known:}

- Variceal bleeding is a common complication of advanced liver disease

- The Baveno VI criteria propose that patients with platelets (PLT) $>150,000$ and liver stiffness measurement $<20 \mathrm{kPa}$ exhibit a very low risk of having high-risk varices (HRVs); they can therefore safely avoid screening endoscopy

- There are ongoing efforts to improve upon the Baveno criteria, in order to further reduce the number of endoscopies

\section{What the new findings are:}

- The Baveno VI criteria were validated in our study population

- A new set of criteria based on the quotient PLT/ $\log _{10}$ (liver stiffness measurement) was developed

- The new set of criteria proved effective at distinguishing patients without HRVs

Jangouk et al showed that by stepwise analysis using a PLT cutoff of $150,000 / \mu \mathrm{L}$ and a model for end-stage liver disease score cutoff of $6,100 \%$ of qualified patients did not have HRVs in screening EGD and the number of endoscopies avoided increased to $54 \%$ of the study population in the US cohort [15]. However, the Italian cohort of the study produced less favorable 
results, with $30 \%$ of the study population avoiding EGD, while $3 \%$ of the patients fulfilling the criteria above did have HRVs.

The value of our criteria is that they combine the liver stiffness and PLT measurements, thus including cases that may be just over the cutoff in the Baveno criteria. Though the use of the binary approach in the Baveno criteria aims to minimize the risk of overlooking HRVs, our study shows that a solitary cutoff, based on continuous variables that combine these data, can be equally safe and may even be more successful in decreasing the number of unnecessary EGDs.

To conclude, our study validates the Baveno VI criteria, contributing to their wider application in clinical practice. The alternative criteria, with the use of the PLT/ $\log _{10}$ LSM ratio we propose, seem to offer even more in terms of the number of endoscopies avoided, while maintaining a very low percentage in terms of missed HRVs. A more extensive study to determine a cutoff level that would provide acceptable percentages of both missed HRVs and avoided endoscopies could provide a valuable alternative to the Baveno VI criteria, or even replace them.

\section{References}

1. de Franchis R; Baveno V Faculty. Revising consensus in portal hypertension: report of the Baveno $\mathrm{V}$ consensus workshop on methodology of diagnosis and therapy in portal hypertension. J Hepatol 2010;53:762-768.

2. Abraldes JG, Bureau C, Stefanescu H, et al; Anticipate Investigators. Noninvasive tools and risk of clinically significant portal hypertension and varices in compensated cirrhosis: The "Anticipate" study. Hepatology 2016;64:2173-2184.

3. Colecchia A, Montrone L, Scaioli E, et al. Measurement of spleen stiffness to evaluate portal hypertension and the presence of esophageal varices in patients with HCV-related cirrhosis. Gastroenterology 2012;143:646-654.

4. Berzigotti A, Seijo S, Arena U, et al. Elastography, spleen size, and platelet count identify portal hypertension in patients with compensated cirrhosis. Gastroenterology 2013;144:102-111.

5. Ding NS, Nguyen T, Iser DM, et al. Liver stiffness plus platelet count can be used to exclude high-risk oesophageal varices. Liver
Int 2016;36:240-245.

6. Stefanescu H, Grigorescu M, Lupsor M, Procopet B, Maniu A, Badea R. Spleen stiffness measurement using Fibroscan for the noninvasive assessment of esophageal varices in liver cirrhosis patients. J Gastroenterol Hepatol 2011;26:164-170.

7. de Franchis R; Baveno VI Faculty. Expanding consensus in portal hypertension: Report of the Baveno VI Consensus Workshop: Stratifying risk and individualizing care for portal hypertension. J Hepatol 2015;63:743-752.

8. Augustin S, Pons M, Maurice JB, et al. Expanding the Baveno VI criteria for the screening of varices in patients with compensated advanced chronic liver disease. Hepatology 2017;66:1980-1988.

9. World Medical Association. World Medical Association Declaration of Helsinki. JAMA 2013;310:2191.

10. European Association for Study of Liver; Asociacion Latinoamericana para el Estudio del Higado. EASL-ALEH Clinical Practice Guidelines: Non-invasive tests for evaluation of liver disease severity and prognosis. J Hepatol 2015;63:237-264.

11. Singh S, Muir AJ, Dieterich DT, Falck-Ytter YT. American Gastroenterological Association Institute Technical Review on the Role of Elastography in Chronic Liver Diseases. Gastroenterology 2017;152:1544-1577.

12. Tapper EB, Castera L, Afdhal NH. FibroScan (vibration-controlled transient elastography): where does it stand in the United States practice. Clin Gastroenterol Hepatol 2015;13:27-36.

13. Bae J, Sinn DH, Kang W, et al. Validation of the Baveno VI and the expanded Baveno VI criteria to identify patients who could avoid screening endoscopy. Liver Int 2018;38:1442-1448.

14. Maurice JB, Brodkin E, Arnold F, et al. Validation of the Baveno VI criteria to identify low risk cirrhotic patients not requiring endoscopic surveillance for varices. J Hepatol 2016;65:899-905.

15. Jangouk P, Turco L, De Oliveira A, Schepis F, Villa E, GarciaTsao G. Validating, deconstructing and refining Baveno criteria for ruling out high-risk varices in patients with compensated cirrhosis. Liver Int 2017;37:1177-1183.

16. Marot A, Trépo E, Doerig C, Schoepfer A, Moreno C, Deltenre P. Liver stiffness and platelet count for identifying patients with compensated liver disease at low risk of variceal bleeding. Liver Int 2017;37:707-716.

17. Augustin S, Pons M, Santos B, Ventura M, Genescà J. Identifying compensated advanced chronic liver disease: when (not) to start screening for varices and clinically significant portal hypertension. In: de Franchis R (editor): Portal hypertension VI. Springer International Publishing: Cham; 2016, pp. 39-49. 